\title{
CONSTRUCTION OF PATHOLOGICAL GÂTEAUX DIFFERENTIABLE FUNCTIONS
}

\author{
ROBERT DEVILLE, MILEN IVANOV, AND SEBASTIÁN LAJARA \\ (Communicated by Thomas Schlumprecht)
}

Abstract. We prove that for many pairs $(X, Y)$ of classical Banach spaces, there exists a bounded, Lipschitz, Gâteaux differentiable function from $X$ to $Y$ whose derivatives are all far apart.

\section{INTRODUCTION}

Let $F$ be a function between real Banach spaces $X$ and $Y$. We say that $F$ has the jump property if $F$ is Gâteaux differentiable at every point of $X$ and there exists a constant $\alpha>0$ such that

$$
\left\|F^{\prime}(x)-F^{\prime}(y)\right\| \geq \alpha \text { whenever } x, y \in X \text { and } x \neq y .
$$

We say that the couple $(X, Y)$ has the jump property if there exists a Lipschitz continuous, bounded function $F: X \longrightarrow Y$ with the jump property.

This concept was first considered by Deville and Hájek in 9], where it was shown that the couple $(X, \mathbb{R})$ never has the jump property and that such a property cannot be achieved if we replace Gâteaux by Fréchet differentiability. There, it was also proved that $\left(\ell^{1}, \mathbb{R}^{2}\right)$ has the jump property and that if $1 \leq p, q<\infty$, then $\left(\ell^{p}, \ell^{q}\right)$ enjoys it if and only if $p \leq q$. Later on, Bayart [5] proved that if $X$ is any separable infinite dimensional Banach space, then $\left(X, c_{0}\right)$ has the jump property.

Notice that a couple of Banach spaces $(X, Y)$ has the jump property if and only if there exists a Lipschitz continuous, bounded and Gâteaux differentiable function $F: X \longrightarrow Y$ such that $\left\|F^{\prime}(x)-F^{\prime}(y)\right\| \geq 1$ whenever $x$ and $y$ are different elements of $X$. It is also clear that if the couple $(X, Y)$ has the jump property, then the space $\mathcal{L}(X, Y)$ of bounded linear operators from $X$ into $Y$ is nonseparable, and that if $Z$ is a Banach space that contains an isomorphic copy of $Y$, then the couple $(X, Z)$ has the jump property as well.

A rather opposite kind of construction was provided in [3], where it was shown that if $X$ and $Y$ are separable Banach spaces, then there exists a continuous Gâteaux differentiable function $F: X \longrightarrow Y$ such that $F^{\prime}(X)=\mathcal{L}(X, Y)$. Some more results in this direction, in the case of Fréchet differentiability, were obtained in [4, 6], [10] and [11.

Received by the editors July 10, 2012 and, in revised form, January 14, 2013 and February 13, 2013.

2010 Mathematics Subject Classification. Primary 46B20, 46G05; Secondary 46T20.

The second author was partially supported by NIS-SU, contract No. 133/2012.

The third author was partialy supported by MTM2011-25377 (Ministerio de Ciencia e Innovación) and by JCCM PEII11-0132-7661. 
Our main aim in this work is to show that the existence of two operators from $X$ to $Y$ of a given form is a sufficient condition to ensure that the couple $(X, Y)$ has the jump property. This condition, which is formulated in terms of the behavior of an unconditional basic sequence in $Y$ with respect to a biorthogonal system in $X$, can be applied whenever $X$ and $Y$ are classical Banach spaces, such as spaces of continuous functions on a compact metric space, Orlicz sequence spaces or $L^{p}$ spaces. We shall also see that such a construction is possible in less classical spaces. Finally, we shall prove that, in some cases, the set of functions between $X$ and $Y$ that enjoy the jump property is lineable. We notice that in all our examples, the space $X$ is separable.

Let us recall some notation and terminology. The symbols $S_{X}$ and $X^{*}$ stand for the unit sphere and the topological dual of a Banach space $X$, respectively. The letter $\mathbb{N}$ denotes the set of positive integers. A Schauder basis $\left(e_{n}\right)_{n}$ in $X$ is said to be unconditional if for every $x \in X$, its expansion $\sum_{n=1}^{\infty} x_{n} e_{n}$ converges unconditionally or, equivalently, if there is a constant $C>0$ such that

$$
\left\|\sum_{n=1}^{\infty} a_{n} x_{n} e_{n}\right\| \leq C\|a\|_{\infty}\left\|\sum_{n=1}^{\infty} x_{n} e_{n}\right\| \text { whenever } a=\left(a_{n}\right)_{n} \in \ell^{\infty} .
$$

A sequence $\left(e_{n}, e_{n}^{*}\right)_{n} \subset X \times X^{*}$ is called a biorthogonal system of $X$ if $e_{i}^{*}\left(e_{j}\right)=\delta_{i j}$ for every $i, j \in \mathbb{N}$. The biorthgonal system is called total if the sequence $\left(e_{n}^{*}\right)_{n}$ separates the points of $X$, i.e., if $x=0$ whenever $e_{n}^{*}(x)=0$ for all $n \in \mathbb{N}$. If $\sup _{n}\left\|e_{n}\right\|<\infty$ and $\sup _{n}\left\|e_{n}^{*}\right\|<\infty$, then we say that the biorthogonal system is bounded. We refer to [8] and [12 for some unexplained notions and basic properties of differentibility and Schauder bases in Banach spaces, respectively.

\section{MAIN THEOREM}

The main result of this paper reads as follows.

Theorem 2.1. Let $X$ and $Y$ be Banach spaces. Suppose that there exist a total, bounded biorthogonal system $\left(e_{n}, e_{n}^{*}\right)_{n} \subset X \times X^{*}$ and an unconditional basic sequence $\left(f_{n}\right)_{n} \subset Y$ such that $\inf _{n}\left\|f_{n}\right\|>0$ and for each $h \in X$, the series $\sum_{n=1}^{\infty} e_{n}^{*}(h) f_{2 n-1}$ and $\sum_{n=1}^{\infty} e_{n}^{*}(h) f_{2 n}$ converge in norm. Then the couple $(X, Y)$ has the jump property.

Let us observe that, under the above assumptions, if

$$
L(h)=\sum_{n=1}^{\infty} e_{n}^{*}(h)\left(f_{2 n-1}+f_{2 n}\right),
$$

then, according to the uniform boundedness principle, $L$ is a bounded linear operator from $X$ into $Y$.

We also notice that the hypotheses of Theorem 2.1 imply that the space $\mathcal{L}(X, Y)$ contains an isomorphic copy of $\ell^{\infty}$ (see the remark after the proof of Theorem 2.1). In particular, $\mathcal{L}(X, Y)$ is nonseparable.

An important tool in our construction of functions with the jump property is the following result, which generalizes [9, Lemma 3].

Lemma 2.2. For each norm $\|\mid \cdot\|$ on $\mathbb{R}^{2}$, each $p=(q, r) \in \mathbb{R}^{2}$ with $q<r$ and each $\varepsilon>0$, there exists a continuously differentiable function $\varphi=\varphi_{p, \varepsilon}: \mathbb{R}^{2} \longrightarrow \mathbb{R}^{2}$ such that:

(i) $\|\mid \varphi(s, t)\| \leq \varepsilon$ for all $(s, t) \in \mathbb{R}^{2}$, 
(ii) $\varphi(s, t)=0$ whenever $s<q$,

(iii) $\left\|\left|\frac{\partial \varphi}{\partial s}(s, t) \|\right| \leq \varepsilon\right.$ for all $(s, t) \in \mathbb{R}^{2}$,

(iv) $\left\|\left|\frac{\partial \varphi}{\partial t}(s, t) \|\right| \leq 1\right.$ for all $(s, t) \in \mathbb{R}^{2}$, and

(v) $\left\|\mid \frac{\partial \varphi}{\partial t}(s, t)\right\| \|=1$ whenever $s \geq r$.

Proof. The function

$$
f(\alpha)=\frac{1}{\|(-\sin \alpha, \cos \alpha)\| \mid}, \quad \alpha \in \mathbb{R},
$$

is clearly Lipschitz on $\mathbb{R}$ and satisfies $c \leq f(\alpha) \leq C$ for some constants $C>c>0$ and all $\alpha \in \mathbb{R}$. Thus, according to the Cauchy-Picard theorem, there exists a (unique) continuously differentiable function $\alpha: \mathbb{R} \longrightarrow \mathbb{R}$ such that

$$
\alpha^{\prime}(t)=\frac{1}{\|(-\sin \alpha(t), \cos \alpha(t))\|} \quad \text { and } \quad \alpha(0)=0 .
$$

In particular, the mapping $\gamma: \mathbb{R} \longrightarrow \mathbb{R}^{2}$ defined by the formula

$$
\gamma(t)=(\cos \alpha(t), \sin \alpha(t)), \quad t \in \mathbb{R},
$$

satisfies $\left|\left\|\gamma^{\prime}(t)\right\|\right|=1$ for all $t \in \mathbb{R}$.

Let $\beta: \mathbb{R} \longrightarrow \mathbb{R}$ be an infinitely differentiable function such that $0 \leq \beta(s) \leq 1$, $\beta(s)=0$ for $s \leq q$ and $\beta(s)=1$ whenever $s \geq r$. If $n>C \varepsilon^{-1}\left(\left\|\beta^{\prime}\right\|_{\infty}+1\right)$, then the function

$$
\varphi(s, t)=\frac{\beta(s)}{n} \gamma(n t)
$$

satisfies the required properties.

Apart from the former lemma, we shall need the following criterion of differentiability, whose proof is left to the reader.

Lemma 2.3. Let $\left(F_{n}\right)_{n}$ be a sequence of Gâteaux differentiable mappings between Banach spaces $X$ and $Y$ satisfying the following properties:

(1) The series $\sum_{n=1}^{\infty} F_{n}$ converges pointwise to a function $F: X \longrightarrow Y$.

(2) For each $h \in X$, the series $\sum_{n=1}^{\infty} F_{n}^{\prime}(x)(h)$ converges uniformly with respect to $x \in X$, and for all $n \in \mathbb{N}$ and $h \in X$, the mapping $X \ni x \mapsto F_{n}^{\prime}(x)(h)$ is continuous.

Then $F$ is Gâteaux differentiable on $X$, and for every $x, h \in X$ we have

$$
F^{\prime}(x)(h)=\sum_{n=1}^{\infty} F_{n}^{\prime}(x)(h) .
$$

Proof of Theorem 2.1. We divide the proof into several steps.

Step 1: Construction of the function $F$. The unconditionality of $\left(f_{n}\right)_{n}$ yields a constant $C>0$ such that

$$
\left\|\sum_{n=1}^{\infty} a_{n} x_{n} f_{n}\right\| \leq C\|a\|_{\infty}\left\|\sum_{n=1}^{\infty} x_{n} f_{n}\right\|
$$

whenever $a=\left(a_{n}\right)_{n} \in \ell^{\infty}$ and $\left(x_{n}\right)_{n} \subset \mathbb{R}$ is any sequence such that the series $\sum_{n=1}^{\infty} x_{n} f_{n}$ is norm convergent.

Since, by the hypothesis, the sequence $\left(1 /\left\|f_{n}\right\|\right)_{n}$ is bounded, thanks to the former inequality we have that for each $h \in X$, the series $\sum_{n=1}^{\infty} e_{n}^{*}(h) \frac{f_{2 n-1}}{\left\|f_{2 n-1}\right\|}$ and $\sum_{n=1}^{\infty} e_{n}^{*}(h) \frac{f_{2 n}}{\left\|f_{2 n}\right\|}$ are norm convergent. Thus, we may assume that $\left\|f_{n}\right\|=1$ for 
all $n \in \mathbb{N}$. Let us write $\mathbb{P}=\left\{(q, r) \in \mathbb{Q}^{2}: q<r\right\}$ and let $k \mapsto\left(n_{k},\left(q_{k}, r_{k}\right)\right)$ be a bijection from $\mathbb{N}$ onto $\mathbb{N} \times \mathbb{P}$ such that $n_{k} \neq k$ for all $k \in \mathbb{N}$. Let $M \geq 1$ be such that $\left\|e_{n}\right\| \leq M$ and $\left\|e_{n}^{*}\right\| \leq M$ for all $n \in \mathbb{N}$, fix $\varepsilon \in(0,1)$ and let $\left(\varepsilon_{k}\right)_{k}$ be any sequence of positive numbers such that $\sum_{k=1}^{\infty} \varepsilon_{k}=\varepsilon / 2 M$. For each $k \in \mathbb{N}$, the formula

$$
\|(s, t)\|_{k}=\left\|t f_{2 k-1}+s f_{2 k}\right\|, \quad(s, t) \in \mathbb{R}^{2},
$$

defines a norm on $\mathbb{R}^{2}$, and bearing in mind that $\left\|f_{n}\right\|=1$ for all $n \in \mathbb{N}$, from (2.1) we obtain

$$
\frac{1}{C} \max \{|s|,|t|\} \leq\|(s, t)\|_{k}, \text { for all }(s, t) \in \mathbb{R}^{2} .
$$

Applying Lemma 2.2 we get a continuously differentiable function $\varphi_{k}: \mathbb{R}^{2} \longrightarrow \mathbb{R}^{2}$ such that

$$
\begin{aligned}
& \left\|\varphi_{k}(s, t)\right\|_{k} \leq \varepsilon_{k} \text { for all }(s, t) \in \mathbb{R}^{2}, \\
& \varphi_{k}(s, t)=0 \text { whenever } s<q_{k}, \\
& \left\|\frac{\partial \varphi_{k}}{\partial s}(s, t)\right\|_{k} \leq \varepsilon_{k} \text { for all }(s, t) \in \mathbb{R}^{2}, \\
& \left\|\frac{\partial \varphi_{k}}{\partial t}(s, t)\right\|_{k} \leq 1 \text { for all }(s, t) \in \mathbb{R}^{2}, \text { and } \\
& \left\|\frac{\partial \varphi_{k}}{\partial t}(s, t)\right\|_{k}=1 \text { whenever } s \geq r_{k} .
\end{aligned}
$$

Let us denote

$$
\begin{gathered}
z_{k}(x)=\left(e_{n_{k}}^{*}(x), e_{k}^{*}(x)\right), \quad x \in X, \\
i_{k}(s, t)=t f_{2 k-1}+s f_{2 k}, \quad(s, t) \in \mathbb{R}^{2},
\end{gathered}
$$

and

$$
F_{k}=i_{k} \circ \varphi_{k} \circ z_{k} .
$$

It is clear that $F_{k}$ is a continuously differentiable function from $X$ into $Y$, and because of (2.3) we have $\left\|F_{k}(x)\right\| \leq \varepsilon_{k}$ for all $x \in X$. Since the series $\sum_{k=1}^{\infty} \varepsilon_{k}$ converges, the formula

$$
F(x)=\sum_{k=1}^{\infty} F_{k}(x), \quad x \in X,
$$

defines a continuous, bounded function from $X$ into $Y$.

Step 2: $F$ is Gâteaux differentiable and Lipschitz. Let us fix $h \in X$. According to Lemma 2.3. it is enough to prove that the series $\sum_{k=1}^{\infty} F_{k}^{\prime}(x)(h)$ converges uniformly with respect to $x$. An easy computation shows that

$$
F_{k}^{\prime}(x)(h)=e_{k}^{*}(h) i_{k}\left(\frac{\partial \varphi_{k}}{\partial t}\left(z_{k}(x)\right)\right)+e_{n_{k}}^{*}(h) i_{k}\left(\frac{\partial \varphi_{k}}{\partial s}\left(z_{k}(x)\right)\right)
$$

for all $k \in \mathbb{N}$ and $x \in X$.

For the sake of brevity we write $\frac{\partial \varphi_{k}}{\partial t}\left(z_{k}(x)\right)=\left(a_{2 k}(x), a_{2 k-1}(x)\right)$. By (2.6) and (2.2) we have $1 \geq\left\|\left(a_{2 k}(x), a_{2 k-1}(x)\right)\right\|_{k} \geq \frac{1}{C} \max \left\{\left|a_{2 k}(x)\right|,\left|a_{2 k-1}(x)\right|\right\}$. Thus, $\left|a_{k}(x)\right| \leq C$ for every $x \in X$ and every $k \in \mathbb{N}$, i.e., $\left\|\left(a_{k}(x)\right)_{k}\right\|_{\infty} \leq C$ for every $x \in X$. Then, thanks to (2.1), for every $h \in X$ and every $N \in \mathbb{N}$ we have

$$
\sup _{x \in X}\left\|\sum_{n=N}^{\infty} e_{n}^{*}(h)\left(a_{2 n}(x) f_{2 n}+a_{2 n-1}(x) f_{2 n-1}\right)\right\| \leq C^{2}\left\|\sum_{n=N}^{\infty} e_{n}^{*}(h)\left(f_{2 n}+f_{2 n-1}\right)\right\| .
$$


Since, by assumption, the series $\sum_{n=1}^{\infty} e_{n}^{*}(h)\left(f_{2 n}+f_{2 n-1}\right)$ is convergent, for every $h \in X$ we have

$$
\sup _{x \in X}\left\|\sum_{n=N}^{\infty} e_{n}^{*}(h)\left(a_{2 n}(x) f_{2 n}+a_{2 n-1}(x) f_{2 n-1}\right)\right\| \rightarrow 0
$$

as $N \rightarrow \infty$. We thus have proved that for every $h \in X$ the series

$$
\sum_{k=1}^{\infty} e_{k}^{*}(h) i_{k}\left(\frac{\partial \varphi_{k}}{\partial t}\left(z_{k}(x)\right)\right)
$$

converges uniformly for $x \in X$.

On the other hand, thanks to inequality (2.5) we have

$$
\left\|e_{n_{k}}^{*}(h) i_{k}\left(\frac{\partial \varphi_{k}}{\partial s}\left(z_{k}(x)\right)\right)\right\| \leq\left\|e_{n_{k}}^{*}\right\|\|h\|\left\|\frac{\partial \varphi_{k}}{\partial s}\left(z_{k}(x)\right)\right\|_{k} \leq M \varepsilon_{k}\|h\|,
$$

and hence the series $\sum_{k=1}^{\infty} e_{n_{k}}^{*}(h) i_{k}\left(\frac{\partial \varphi_{k}}{\partial s}\left(z_{k}(x)\right)\right)$ converges uniformly with respect to $x$ as well. Therefore, according to Lemma 2.3, $F$ is Gâteaux differentiable at every point of $X$, and

$$
F^{\prime}(x)(h)=\sum_{k=1}^{\infty} F_{k}^{\prime}(x)(h) \quad \text { whenever } \quad x, h \in X .
$$

Bearing in mind that $\left|a_{k}(x)\right| \leq C$ for all $k \in \mathbb{N}$, from inequalities (2.1) and (2.9) (with $N=1$ ), for all $h \in X$ we have

$$
\sup _{x \in X}\left\|\sum_{k=1}^{\infty} e_{k}^{*}(h) i_{k}\left(\frac{\partial \varphi_{k}}{\partial t}\left(z_{k}(x)\right)\right)\right\| \leq C^{2}\|L\|\|h\|,
$$

where $L$ is the bounded linear operator defined just after the statement of Theorem 2.1. This inequality and (2.10) imply

$$
\sup _{x \in X}\left\|F^{\prime}(x)\right\| \leq C^{2}\|L\|+\varepsilon / 2 .
$$

Thus, the function $F$ is Lipschitz on $X$.

Step 3: $F$ has the jump property. Take any two vectors $x, y \in X$ such that $x \neq y$. Pick $m \in \mathbb{N}$ such that $e_{m}^{*}(x) \neq e_{m}^{*}(y)$. We can assume that $e_{m}^{*}(x)<e_{m}^{*}(y)$. Find $q, r \in \mathbb{Q}$ so that $e_{m}^{*}(x)<q<r<e_{m}^{*}(y)$. Find a natural number $k$ such that $n_{k}=m, q_{k}=q$ and $r_{k}=r$. Since $\varphi_{k}(s, t)=0$ whenever $s<q_{k}$, according to (2.4) we have $\frac{\partial \varphi_{k}}{\partial t}\left(z_{k}(x)\right)=\frac{\partial \varphi_{k}}{\partial t}\left(e_{n_{k}}^{*}(x), e_{k}^{*}(x)\right)=0$, and using (2.8) with $h=e_{k}$ we get $F_{k}^{\prime}(x)\left(e_{k}\right)=0$. On the other hand, from (2.7) we obtain $\left\|\frac{\partial \varphi_{k}}{\partial t}\left(e_{n_{k}}^{*}(y), e_{k}^{*}(y)\right)\right\|_{k}=1$. Consequently, $\left\|F_{k}^{\prime}(y)\left(e_{k}\right)\right\|=1$, and thus,

$$
\left\|F_{k}^{\prime}(x)\left(e_{k}\right)-F_{k}^{\prime}(y)\left(e_{k}\right)\right\|=1 .
$$

Moreover, from (2.8) and (2.5) it follows that

$$
\left\|F_{j}^{\prime}(x)\left(e_{k}\right)\right\| \leq \varepsilon_{k} \text { and }\left\|F_{j}^{\prime}(y)\left(e_{k}\right)\right\| \leq \varepsilon_{k} \text { for all } j \neq k \text {. }
$$

Therefore,

$$
\left\|F^{\prime}(x)-F^{\prime}(y)\right\| \geq M^{-1}\left\|F^{\prime}(x)\left(e_{k}\right)-F^{\prime}(y)\left(e_{k}\right)\right\| \geq M^{-1}\left(1-2 \sum_{j \neq k} \varepsilon_{j}\right)>M^{-1}(1-\varepsilon),
$$

as we wanted to show. 
Remark 2.4. Under the assumptions of Theorem 2.1 it follows that for every $a=$ $\left(a_{n}\right)_{n} \in \ell^{\infty}$ the formula

$$
L_{a}(h)=\sum_{n=1}^{\infty} e_{n}^{*}(h)\left(a_{2 n} f_{2 n}+a_{2 n-1} f_{2 n-1}\right), \quad h \in X,
$$

defines a bounded linear operator from $X$ into $Y$. More precisely, there exist constants $C_{1}, C_{2}>0$ such that

$$
C_{1}\|a\|_{\infty} \leq\left\|L_{a}\right\| \leq C_{2}\|a\|_{\infty} .
$$

Indeed, thanks to inequality (2.1) we have

$$
\left\|L_{a}(h)\right\| \leq C\|a\|_{\infty}\left\|\sum_{n=1}^{\infty} e_{n}^{*}(h)\left(f_{2 n}+f_{2 n-1}\right)\right\| \leq C\|a\|_{\infty}\|L\|\|h\|
$$

for every $h \in X$ and some constant $C>0$. (Here, $L$ denotes the bounded linear operator defined after the statement of Theorem 2.1)

On the other hand (assuming that $\left\|f_{n}\right\|=1$ for each $n \in \mathbb{N}$ ), if we denote $M=\sup \left\{\left\|e_{1}\right\|,\left\|e_{2}\right\|, \ldots\right\}$, from inequality (2.2) we get

$$
\left\|L_{a}\right\| \geq \frac{1}{M}\left\|L_{a}\left(e_{m}\right)\right\|=\frac{1}{M}\left\|a_{2 m-1} f_{2 m-1}+a_{2 m} f_{2 m}\right\| \geq \frac{1}{M C} \max \left\{\left|a_{2 m-1}\right|,\left|a_{2 m}\right|\right\}
$$

for every $m \in \mathbb{N}$. Hence, $\left\|L_{a}\right\| \geq \frac{1}{M C}\|a\|_{\infty}$ for every $a \in \ell^{\infty}$.

As a consequence of (2.11) it follows that the space $\ell^{\infty}$ embeds into $\mathcal{L}(X, Y)$. Thus, our theorem does not apply if the space $\mathcal{L}(X, Y)$ does not contain any isomorphic copy of $\ell^{\infty}$. It is not known whether the couple $\left(X, \mathbb{R}^{2}\right)$ has the jump property whenever $X$ is a separable Banach space with a nonseparable dual. An affirmative answer was given in 9 whenever $X=\ell^{1}$, but the question remains open if $X$ is the James tree space. In this case, $\mathcal{L}\left(X, \mathbb{R}^{2}\right)$ does not contain any isomorphic copy of $\ell^{\infty}$.

\section{Application to classical Banach spaces}

As a particular case of Theorem 2.1, we obtain the aforementioned result by Bayart in [5].

Corollary 3.1. If $X$ is a separable Banach space, then the couple $\left(X, c_{0}\right)$ has the jump property. In particular, if $K$ is an infinite metric compact space, then $(X, \mathcal{C}(K))$ has the jump property.

Proof. It is well-known (see e.g. [12, Theorem 1.f.14]) that there exists a bounded, total biorthogonal system $\left(e_{n}, e_{n}^{*}\right)_{n} \subset X \times X^{*}$ such that $\left\|e_{n}^{*}\right\|=1$ for all $n \in \mathbb{N}$, and $X=\overline{\operatorname{span}\left(e_{n}\right)_{n}}$. In particular, for every $h \in X$ we have $\lim _{n} e_{n}^{*}(h)=0$, that is, $\left(e_{n}^{*}(h)\right)_{n} \in c_{0}$, and if we denote by $\left(f_{n}\right)_{n}$ the unit vector basis of $c_{0}$, then

$$
\left\|\sum_{n=1}^{\infty} e_{n}^{*}(h) f_{2 n-1}\right\| \leq\|h\| \text { and }\left\|\sum_{n=1}^{\infty} e_{n}^{*}(h) f_{2 n}\right\| \leq\|h\| .
$$

Since $\left(f_{n}\right)_{n}$ is unconditional and normalized, from Theorem 2.1 we deduce that the couple $\left(X, c_{0}\right)$ has the jump property. The second statement follows from the fact that $\mathcal{C}(K)$ contains an isomorphic copy of $c_{0}$. 
A Schauder basis of a Banach space is called subsymmetric (cf. [12, p. 114]) if it is unconditional and equivalent to any of its subsequences. It is well-known (see e.g. [12, Proposition 3.a.3]) that a Schauder basis $\left(e_{n}\right)_{n}$ is subsymmetric if it is symmetric; that is, if for any permutation $\pi: \mathbb{N} \rightarrow \mathbb{N},\left(e_{n}\right)_{n}$ is equivalent to $\left(e_{\pi(n)}\right)_{n}$ (cf. [12, p. 113]).

Corollary 3.2. Let $X$ be a Banach space with a bounded Schauder basis $\left(e_{n}\right)_{n}$, let $Y$ be a Banach space, and assume there exists $U \in \mathcal{L}(X, Y)$ such that $\left(U\left(e_{n}\right)\right)_{n}$ is a subsymmetric basic sequence in $Y$ with $\inf _{n}\left\|U\left(e_{n}\right)\right\|>0$. Then $(X, Y)$ has the jump property.

Proof. Observe that if $\left(e_{n}^{*}\right)_{n}$ is the sequence of biorthogonal functionals to $\left(e_{n}\right)_{n}$ and if we denote $f_{n}=U\left(e_{n}\right)$, then $U(h)=\sum_{n=1}^{\infty} e_{n}^{*}(h) f_{n}$ for all $h \in X$.

On the other hand, thanks to the subsymmetry of $\left(f_{n}\right)_{n}$, the sequences $\left(f_{2 n}\right)_{n}$ and $\left(f_{2 n-1}\right)_{n}$ are equivalent to $\left(f_{n}\right)_{n}$. Thus, the series $\sum_{n=1}^{\infty} e_{n}^{*}(h) f_{2 n-1}$ and $\sum_{n=1}^{\infty} e_{n}^{*}(h) f_{2 n}$ converge for all $h \in X$. Since the basic sequence $\left(f_{n}\right)_{n}$ is unconditional and satisfies $\inf _{n}\left\|f_{n}\right\|>0$, we can apply Theorem 2.1

The former corollary implies that if $X$ has a subsymmetric basis (in particular, if $X$ has a symmetric basis), then the couple $(X, X)$ satisfies the jump property.

Corollary 3.3. If $\infty>q \geq p \geq 1$, then the couple $\left(\ell^{p}, \ell^{q}\right)$ has the jump property. More generally, let $M$ and $N$ be two Orlicz functions such that $N(t) \leq k_{1} M\left(k_{2} t\right)$ for some constants $k_{1}, k_{2}>0$ and all $t$ in a neighbourhood of zero. If $h_{M}$ and $h_{N}$ are the corresponding Orlicz sequence spaces, then the couple $\left(h_{M}, h_{N}\right)$ has the jump property.

Proof. It is clear that the unit vector basis of $h_{N}$ is symmetric. On the other hand, using an argument as in [12, Proposition 4.a.5], from the inequality $N(t) \leq$ $k_{1} M\left(k_{2} t\right)$ we deduce that the inclusion mapping $U: h_{M} \longrightarrow h_{N}$ is well-defined, linear and continuous. Thus, Corollary 3.2 applies.

The first assertion in the former corollary, which is a consequence of the result on the Orlicz sequence spaces, is [9, Theorem 3]. There it was also shown that $\left(\ell^{p}, \ell^{q}\right)$ does not have jump property when $p>q$, using the fact that in this case the space $\mathcal{L}\left(\ell^{p}, \ell^{q}\right)$ is separable.

Another consequence of Theorem 2.1 is the following result.

Corollary 3.4. Let $X$ be a Banach space with a bounded Schauder basis $\left(e_{n}\right)_{n}$, let $Y$ be a Banach space, and assume there exists $U \in \mathcal{L}(X, Y)$ such that:

(1) $\left(U\left(e_{n}\right)\right)_{n}$ is an unconditional basic sequence in $Y$ with $\inf _{n}\left\|U\left(e_{n}\right)\right\|>0$ and

(2) $Y$ is isomorphic to $Y \oplus Y$.

Then $(X, Y)$ has the jump property.

Proof. Let $P$ be an isomorphism from $Y \oplus Y$ onto $Y$, and let us define, for $h \in X$, $T(h)=P(U(h), 0), S(h)=P(0, U(h))$, and for $n \in \mathbb{N}, f_{2 n-1}=T\left(e_{n}\right)$ and $f_{2 n}=$ $S\left(e_{n}\right)$. Clearly, $\left(f_{n}\right)_{n}$ an unconditional basic sequence in $Y\left(\right.$ with $\inf _{n}\left\|f_{n}\right\|>0$ ). 
On the other hand, because of the continuity of $T$ and $S$, for every $h \in X$ we have

$$
T(h)=T\left(\sum_{n=1}^{\infty} e_{n}^{*}(h) e_{n}\right)=\sum_{n=1}^{\infty} e_{n}^{*}(h) f_{2 n-1}
$$

and

$$
S(h)=\sum_{n=1}^{\infty} e_{n}^{*}(h) f_{2 n} .
$$

So, the above series converge and Theorem 2.1 applies.

A particular case of the former corollary is the following result.

Corollary 3.5. If $X$ is a separable Banach space with an unconditional basis and $X$ is isomorphic to $X \oplus X$, then the couple $(X, X)$ has the jump property.

This result applies when $X=L^{p}([0,1])$, for $1<p<\infty$. Indeed, it is wellknown that the space $L^{p}([0,1])$ is isomorphic to its square. On the other hand, if $p \in(1, \infty)$, then the Haar system constitutes an unconditional basis of $L^{p}([0,1])$ (cf. [13, Theorem 2.c.5]). Thus, the couple $\left(L^{p}([0,1]), L^{p}([0,1])\right)$ has the jump property if $1<p<\infty$. As an easy consequence of this fact we get the following result.

Corollary 3.6. If $2 \geq p \geq q \geq 1$ and $p \neq 1$, then the couple $\left(L^{p}([0,1]), L^{q}([0,1])\right)$ has the jump property.

Proof. Since the space $L^{q}([0,1])$ contains an isomorphic copy of $L^{p}([0,1])$ (cf. [1, Prop. 11.1.9]) and $\left(L^{p}([0,1]), L^{p}([0,1])\right)$ has the jump property, the couple $\left(L^{p}([0,1]), L^{q}([0,1])\right)$ also satisfies this property.

Remark 3.7. The couple $\left(L^{2}([0,1]), L^{p}([0,1])\right)$ has the jump property for every $p \geq 1$. This follows from the fact that $L^{2}([0,1])$ is isomorphic to a subspace of $L^{p}([0,1])$ (see e.g. [12, Theorem 2.b.3]). Notice also that for all $1<p, q<\infty$, $\mathcal{L}\left(L^{p}([0,1]), L^{q}([0,1])\right)$ contains an isomorphic copy of $\ell^{\infty}$. This follows easily from the fact that the space generated by the Rademacher functions is complemented both in $L^{p}([0,1])$ and in $L^{q}([0,1])$. Therefore, unlike the case of $\ell^{p}$ spaces, it is not clear to us whether there exist couples $(p, q)$ such that $\left(L^{p}([0,1]), L^{q}([0,1])\right)$ fails to have the jump property. In particular, we do not know if $\left(L^{1}([0,1]), L^{1}([0,1])\right)$ has this property.

We conclude this section with some more couples of Banach spaces satisfying the jump property and another one which fails this property.

Corollary 3.8. If $\mathcal{T}$ is the Tsirelson's space, then the couples $(\mathcal{T}, \mathcal{T})$ and $\left(\mathcal{T}^{*}, \mathcal{T}^{*}\right)$ have the jump property. We notice that the space $\mathcal{T}$ does not have any subsymmetric Schauder basis.

Proof. Let $\left(e_{n}\right)_{n}$ be the sequence of unit vectors of $\mathcal{T}$. According to [7, Propositions I.9 and I.12] it follows that $\left(e_{n}\right)_{n}$ is an unconditional basis of $\mathcal{T}$, which is equivalent to its subsequences $\left(e_{2 n-1}\right)_{n}$ and $\left(e_{2 n}\right)_{n}$. In particular, $\mathcal{T}$ is isomorphic to its square, and we can apply Corollary 3.5. A similar argument shows that the couple $\left(\mathcal{T}^{*}, \mathcal{T}^{*}\right)$ shares this property.

Corollary 3.9. If $J$ is the James space, then the couple $\left(J, \ell^{2}\right)$, and hence $(J, J)$, has the jump property. We observe that the space $J$ does not have any unconditional Schauder basis. 
Proof. Let $\left(u_{n}\right)_{n}$ and $\left(f_{n}\right)_{n}$ be the canonical vector bases of $J$ and $\ell^{2}$, respectively. Let us denote by $\left(s_{n}\right)_{n}$ the summing basis associated to $\left(u_{n}\right)_{n}$. If $e_{n}^{*}=u_{n}^{*}-u_{n+1}^{*}$, then $\left(e_{n}^{*}\right)_{n}$ is the sequence of biorthogonal functionals associated to $\left(s_{n}\right)_{n}$.

Let us define, for each $n \in \mathbb{N}, U\left(s_{n}\right)=f_{n}$, and extend $U$ by linearity. For each $x=\left(x_{n}\right)_{n} \in J$ with finite support we have

$$
\|U(x)\|_{2}^{2}=\sum_{n=1}^{\infty}\left(x_{n+1}-x_{n}\right)^{2} \leq\|x\|_{J}^{2} .
$$

Hence, $U$ extends to a continuous linear operator from $J$ to $\ell^{2}$. As $\left(f_{n}\right)_{n}$ is a symmetric basis of $\ell^{2}$, Corollary 3.2 ensures that the couple $\left(J, \ell^{2}\right)$ has the jump property. Since $\ell^{2}$ is isomorphic to a subspace of $J$, the couple $(J, J)$ also enjoys this property.

Example 3.10. If $X$ is the Banach space constructed by Argyros and Haydon in [2], then $(X, X)$ fails the jump property.

Proof. This space is separable, has the property that every $T \in \mathcal{L}(X)$ is of the form $\lambda I+K$ where $I$ is the identity operator and $K$ is a compact operator, and satisfies that $\mathcal{L}(X)$ is separable. Hence $(X, X)$ does not have the jump property.

\section{THE SET OF FUnCTIONS SATISFYING THE JUMP PROPERTY}

Let $X$ and $Y$ be Banach spaces and let $G(X, Y)$ denote the space of all bounded and Lipschitz functions from $X$ to $Y$ which are Gâteaux differentiable at each point of $X$, endowed with its natural norm

$$
\|F\|:=\sup \{\|F(x)\| ; x \in X\}+\sup \left\{\left\|F^{\prime}(x)\right\| ; x \in X\right\} .
$$

Let us also write $G_{*}(X, Y):=\{f \in G(X, Y) ; f$ has the jump property $\}$. The couple $(X, Y)$ has the jump property if and only if $G_{*}(X, Y) \neq \emptyset$. Bayart [5] has shown that if $X$ is a separable Banach space, then $G_{*}\left(X, c_{0}\right)$ is spaceable; i.e., $G_{*}\left(X, c_{0}\right) \cup\{0\}$ contains a closed infinite dimensional subspace of $G\left(X, c_{0}\right)$. We notice here the following result.

Proposition 4.1. Let $X$ be a Banach space with a bounded Schauder basis, and let $Y$ be a Banach space. Assume that there exists $U \in \mathcal{L}(X, Y)$ such that $\left(U\left(e_{n}\right)\right)_{n}$ is a subsymmetric basic sequence in $Y$ with $\inf _{n}\left\|U\left(e_{n}\right)\right\|>0$. Then $G_{*}(X, Y)$ is lineable; i.e., $G_{*}(X, Y) \cup\{0\}$ contains an infinite dimensional subspace of $G(X, Y)$.

Proof. Let $\left\{I_{p}\right\}_{p \in \mathbb{N}}$ be a partition of $\mathbb{N}$ into countably many infinite subsets, and for each $p \in \mathbb{N}$ put $Y_{p}=\overline{\operatorname{span}\left(U\left(e_{n}\right)\right)_{n \in I_{p}}}$. Let $\pi_{p}: \mathbb{N} \longrightarrow I_{p}$ be an increasing bijection. Since the sequence $\left(U\left(e_{n}\right)\right)_{n}$ is subsymmetric, it is equivalent to the sequence $\left(U\left(e_{\pi_{p}(n)}\right)\right)_{n}$ which itself is subsymmetric. Therefore, for each $p$, if we define $U_{p}\left(e_{n}\right)=U\left(e_{\pi_{p}(n)}\right)$, then $\left(U_{p}\left(e_{n}\right)\right)_{n}$ is a subsymmetric basic sequence in $Y_{p}$ such that $\inf _{n}\left\|U_{p}\left(e_{n}\right)\right\|>0$. Applying Corollary 3.2 to the couple $\left(X, Y_{p}\right)$ we get a Gâteaux differentiable function $F_{p}: X \rightarrow Y_{p}$ such that

$$
\left\|F_{p}^{\prime}(x)-F_{p}^{\prime}(y)\right\|_{\mathcal{L}\left(X, Y_{p}\right)} \geq 1 \text { whenever } x, y \in X \text { and } x \neq y .
$$


Notice that $\bigoplus_{p=1}^{\infty} \mathcal{L}\left(X, Y_{p}\right)=\mathcal{L}\left(X, \bigoplus_{p=1}^{\infty} Y_{p}\right) \subset \mathcal{L}(X, Y)$, so there exists a constant $c>0$ (coming from the unconditionality of $\left.\left(U\left(e_{n}\right)\right)_{n}\right)$ such that, if $T_{p} \in$ $\mathcal{L}\left(X, Y_{p}\right)$ and $T=\sum T_{p} \in \mathcal{L}(X, Y)$, then $\|T\| \geq c \sup \left\{\left\|T_{p}\right\| ; p \in \mathbb{N}\right\}$. We claim that for each $N \in \mathbb{N}$ and each sequence of scalars $\left(\beta_{1}, \ldots, \beta_{N}\right)$ which is not identically equal to zero, the function $F=\sum_{p=1}^{N} \beta_{p} F_{p}$ satisfies the jump property. Indeed, find $p_{0}$ such that $\beta_{p_{0}} \neq 0$. Now, for every $x, y \in X$ with $x \neq y$ we have $F_{p}^{\prime}(x)-F_{p}^{\prime}(y) \in \mathcal{L}\left(X, Y_{p}\right)$ for all $p$, and $F^{\prime}(x)-F^{\prime}(y) \in \bigoplus_{p=1}^{\infty} \mathcal{L}\left(X, Y_{p}\right) \subset \mathcal{L}(X, Y)$. Therefore,

$$
\left\|F^{\prime}(x)-F^{\prime}(y)\right\|=\left\|\sum_{p=1}^{N} \beta_{p}\left(F_{p}^{\prime}(x)-F_{p}^{\prime}(y)\right)\right\| \geq c\left|\beta_{p_{0}}\right|\left\|F_{p_{0}}^{\prime}(x)-F_{p_{0}}^{\prime}(y)\right\| \geq c\left|\beta_{p_{0}}\right|,
$$

as we wanted to show.

We are unable to decide whether, under the assumptions of Proposition 4.1, the set $G_{*}(X, Y)$ is spaceable.

\section{ACKNOWLEDGMENTS}

The authors are very grateful to the anonymous referee for contributions that improved the overall shape of the paper. Thanks are also due to Prof. J. Rodriguez for interesting discussions on the subject of this work.

\section{REFERENCES}

[1] Fernando Albiac and Nigel J. Kalton, Topics in Banach space theory, Graduate Texts in Mathematics, vol. 233, Springer, New York, 2006. MR2192298 (2006h:46005)

[2] Spiros A. Argyros and Richard G. Haydon, A hereditarily indecomposable $\mathcal{L}_{\infty}$-space that solves the scalar-plus-compact problem, Acta Math. 206 (2011), no. 1, 1-54, DOI 10.1007/s11511-011-0058-y. MR2784662(2012e:46031)

[3] D. Azagra, M. Jiménez-Sevilla, and R. Deville, On the range of the derivatives of a smooth function between Banach spaces, Math. Proc. Cambridge Philos. Soc. 134 (2003), no. 1, 163-185, DOI 10.1017/S0305004102006278. MR1937801(2004c:46069)

[4] D. Azagra, M. Fabian, and M. Jiménez-Sevilla, Exact filling of figures with the derivatives of smooth mappings between Banach spaces, Canad. Math. Bull. 48 (2005), no. 4, 481-499, DOI 10.4153/CMB-2005-045-9. MR2176147 (2006f:46011)

[5] Frédéric Bayart, Linearity of sets of strange functions, Michigan Math. J. 53 (2005), no. 2, 291-303, DOI 10.1307/mmj/1123090769. MR2152701 (2006b:46048)

[6] J. M. Borwein, M. Fabian, and P. D. Loewen, The range of the gradient of a Lipschitz $C^{1}$-smooth bump in infinite dimensions, Israel J. Math. 132 (2002), 239-251, DOI 10.1007/BF02784514. MR1952623 (2003j:58011)

[7] Peter G. Casazza and Thaddeus J. Shura, Tsirel'son's space. With an appendix by J. Baker, O. Slotterbeck and R. Aron, Lecture Notes in Mathematics, vol. 1363, Springer-Verlag, Berlin, 1989. MR.981801 (90b:46030)

[8] Robert Deville, Gilles Godefroy, and Václav Zizler, Smoothness and renormings in Banach spaces, Pitman Monographs and Surveys in Pure and Applied Mathematics, vol. 64, Longman Scientific \& Technical, Harlow, 1993. MR1211634 (94d:46012)

[9] Robert Deville and Petr Hájek, On the range of the derivative of Gateaux-smooth functions on separable Banach spaces, Israel J. Math. 145 (2005), 257-269, DOI 10.1007/BF02786693. MR2154729(2007e:46035)

[10] Marián Fabian, Ondřej F. K. Kalenda, and Jan Kolář, Filling analytic sets by the derivatives of $C^{1}$-smooth bumps, Proc. Amer. Math. Soc. 133 (2005), no. 1, 295-303, DOI 10.1090/S00029939-04-07730-5. MR2086222 (2005f:46089)

[11] T. Gaspari, On the range of the derivative of a real-valued function with bounded support, Studia Math. 153 (2002), no. 1, 81-99, DOI 10.4064/sm153-1-6. MR1948929 (2003k:46057) 
[12] Joram Lindenstrauss and Lior Tzafriri, Classical Banach spaces. I. Sequence spaces. Ergebnisse der Mathematik und ihrer Grenzgebiete, vol. 92, Springer-Verlag, Berlin, 1977. MR.0500056 (58 \#17766)

[13] Joram Lindenstrauss and Lior Tzafriri, Classical Banach spaces. II. Function spaces. Ergebnisse der Mathematik und ihrer Grenzgebiete [Results in Mathematics and Related Areas], vol. 97, Springer-Verlag, Berlin, 1979. MR.540367 (81c:46001)

Institut de Mathématiques de Bordeaux, Université Bordeaux 1, 351, Cours De LA Libération, 33400 TAlence, France

E-mail address: Robert.Deville@math.u-bordeaux1.fr

Faculty of Mathematics and Informatics, Sofia University, 5 James Bourchier BouleVARD, 1164 SOFIA, Bulgaria

E-mail address: milen@fmi.uni-sofia.bg

Departamento de Matemáticas, Escuela de Ingenieros Industriales, Universidad de Castilla-La Mancha, Campus Universitario, 02071 Albacete, Spain

E-mail address: Sebastian.Lajara@uclm.es 